\title{
Pulmonary Cavities after Diagnostic Transbronchial Cryobiopsy in a Lung Transplantation Patient
}

\author{
Rebeca Sicilia Torres ${ }^{\mathrm{a}}$ Felipe Rodríguez de Castro ${ }^{\mathrm{a}}$ Carlos Cabrera López $^{\mathrm{a}}$ \\ Gabriel Juliá Serdá ${ }^{a}$ Fernando Rezola Sáez ${ }^{b}$ Rubén Pestana ${ }^{b}$ \\ Departments of a Pulmonary Medicine and ${ }^{b}$ Radiology, University Hospital Doctor Negrín, \\ Las Palmas de Gran Canaria, Spain
}

Over the last few years, cryobiopsy has been accepted as a safe and useful technique in the differential diagnosis of diffuse interstitial lung disease [1]. It has also been successfully used to identify lung transplant rejection with a low complication rate [2]. A 46-year-old woman with a history of primary ciliary dyskinesia and lung transplantation 14 years ago was admitted to the hospital due to lung function decline and a 10-day symptomatology of dyspnea. Physical examination, initial routine laboratory tests and chest radiograph were unremarkable (Fig. 1a). Bronchoalveolar lavage (BAL) was performed during flexible bronchoscopy at the time of admission and prophylactic antimicrobial treatment was started according to protocol while awaiting results. Forty-eight hours after admission, a transbronchial cryobiopsy was carried out via a flexible bronchoscope, under general anesthesia with a laryngeal mask, using fluoroscopy and the Arndt endobronchial blocker. Two samples were obtained from different segments (anterior and lateral) of the left lower lobe with minimal self-limited bleeding without major complications (Fig. 1b). Six days after the procedure, a chest high-resolution computed tomography (HRCT) showed two small cavitary lesions in the left lower lobe surrounded by a halo of ground-glass opacity (Fig. 1c) and air-trapping on expiratory sections. Gram-negative

\section{KARGER}

(c) 2017 S. Karger AG, Basel

E-Mail karger@karger.com

www.karger.com/res bacteria identified as Pseudomonas aeruginosa were isolated from bronchial aspirate. All microbiological tests on peripheral blood and BAL were negative. Histologically, the findings revealed bronchiolitis obliterans confirming the suspected diagnosis of chronic lung transplant rejection. Our patient received high-dose systemic steroid therapy with a good response. Nineteen days following the technique, a HRCT showed almost complete resolution of both lesions (Fig. 1d).

As far as we know, there is no report on the literature describing such images after a cryobiopsy. These changes in the lung parenchyma might occur in all patients and we might not have seen them yet as it is not a common practice to perform a CT after a cryobiopsy. We find the conjunction of the laceration itself, the local effects of congelation around the freezing point and a feasible valve mechanism due to the presence of bronchiolitis obliterans in our patient more suitable for explanation. Pulmonary cavities may be the visible expression of different infectious and noninfectious processes; therefore, to be aware that this procedure may cause cavities in the lung parenchyma may be clinically relevant. Lack of knowledge of these transient injuries might lead to an incorrect diagnosis and delayed steroid treatment in these patients. 


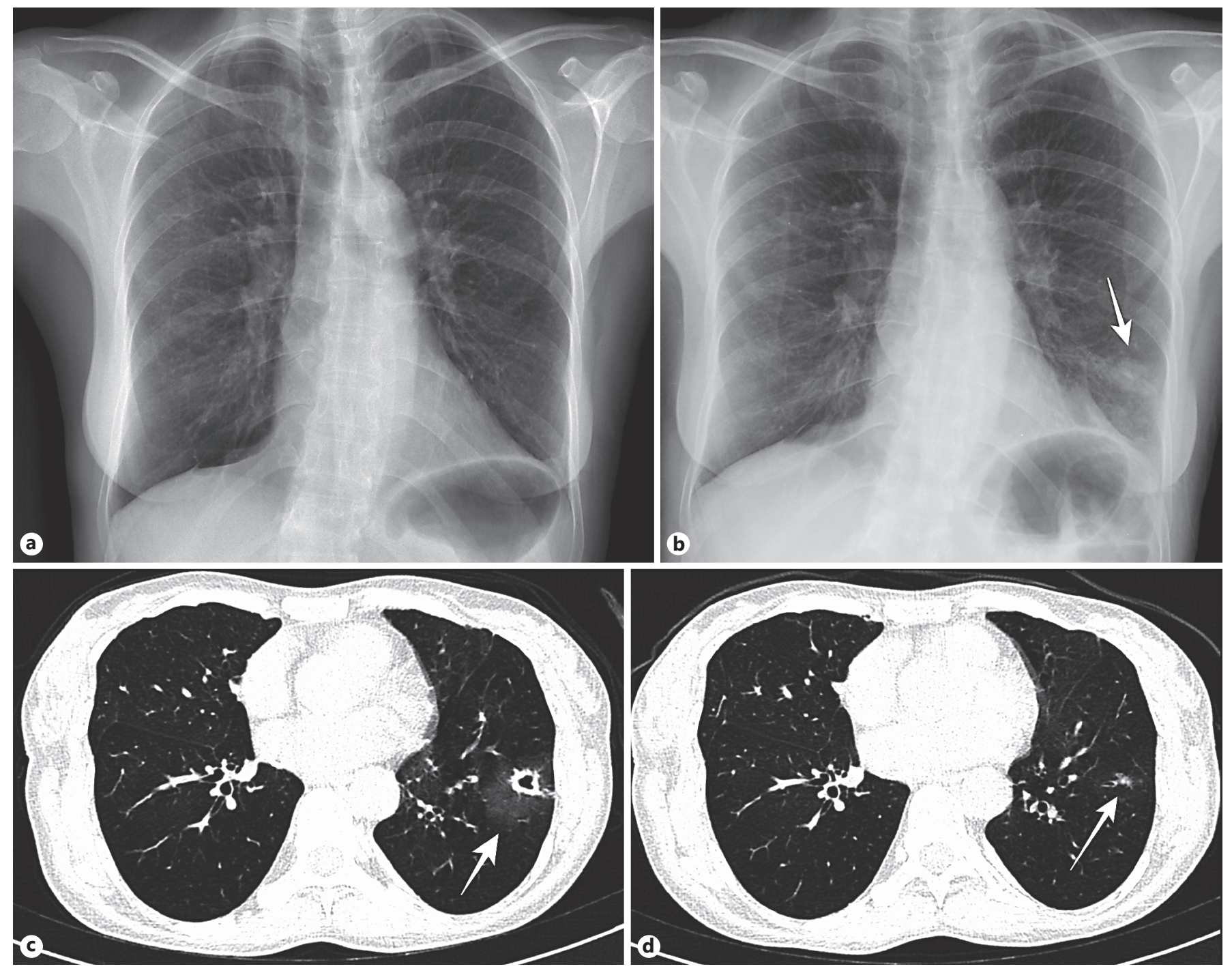

Fig. 1. a Normal chest radiograph at the time of admission. b Chest radiograph a few hours after the cryobiopsy (arrow). c Visualization on HRCT of one left lower lobe cavitary lesion with surrounding ground-glass opacity (arrow) 6 days later. d HRCT shows almost complete resolution of the cavities 19 days after the procedure.

\section{References}

Pulmonary Cavities after Diagnostic Transbronchial Cryobiopsy
1 Bango-Álvarez A, Ariza-Prota M, Torres-Rivas $\mathrm{H}$, Fernández-Fernández L, Prieto A, Sánchez I, Gil M, Pando-Sandoval A: Transbronchial cryobiopsy in interstitial lung disease: experience in 106 cases - how to do it. ERJ Open Res 2017;3:00148-2016.
2 Fruchter O, Fridel L, Rosengarten D, Raviv Y, Rosanov V, Kramer MR: Transbronchial cryo-biopsy in lung transplantation patients: first report. Respirology 2013;18:669-673. 